\title{
Erratum to: TMPRSS2:ERG fusion gene occurs less frequently in Chinese patients with prostate cancer
}

\author{
Hui Jiang ${ }^{1,2}$ - Xueying $\mathrm{Mao}^{3} \cdot$ Xiaoyi Huang ${ }^{1} \cdot$ Jing Zhao $^{1} \cdot$ Lumei Wang $^{4}$. \\ Jingjing $\mathrm{Xu}^{1}$ • Hongwei $\mathrm{Zhang}^{5} \cdot$ Yongjie $\mathrm{Lu}^{3}$ - Yongwei $\mathrm{Yu}^{1}$
}

Published online: 22 July 2016

(C) International Society of Oncology and BioMarkers (ISOBM) 2016

Erratum to: Tumor Biol.

DOI: 10.1007/s13277-016-5116-9

The original version of this article unfortunately contained an error in the author group section.

The supercript of co-author Yongjie Lu should have been 3 not 4 . The corrected author group is shown above.

The online version of the original article can be found at http://dx.doi. org/10.1007/s113277-016-5116-9.

Yongwei Yu

yongweiyu@126.com

1 Department of Pathology, Changhai Hospital, Second Military Medical University, 168 Changhai Road, Shanghai 200433, China

2 Department of Pathophysiology, Second Military Medical University, Shanghai 200433, China

3 Molecular Oncology \& Imaging Centre, Barts and The London School of Medicine and Dentistry, Queen Mary University of London, London, UK

4 Changhai Hospital, Second Military Medical University, Shanghai 200433, China

5 Department of Epidemiology, Second Military Medical University, Shanghai 200433, China 